Arquitectura +

ISSN: 2518-2943

www.revistas.uni.edu.ni/index.php?jo

urnal=arquitectura

\title{
Artículos
}

\section{Viviendas pasivas a favor del medio ambiente} Passive housing for the environment

Ortiz Monroy Héctor / hector.ortiz@uaq.mx

* Quizamán Velasco Estefani Rosalía / equizaman@gmail.com

Maestro en Ciencias en Arquitectura y Urbanismo IPN, México. Neuro-arquitecturamx

* Licenciada en Diseño de Interiores, egresada del Centro Universitario de Comunicación en CDMX, y Maestría en proceso en la Universidad Autónoma de Querétaro área de Arquitectura, experiencia laboral en áreas de diseño, construcción y remodelaciones.

\begin{abstract}
Recently, the energy crisis and the growing environmental pollution have forced us to look for alternative sources to satisfy the habitable and calorific needs of the human being, focusing mainly on the greatest natural energy existing: the sun.

Passive houses use resources of nature for different efficiencies and implement passive or transforming way through technologies that work towards the demands of the people.

The passive constructions are designed and built under parameters established by the Passivhaus with the purpose of offering users living spaces by taking considerable economic savings in the energy amount, offering significant annual savings compared to conventionally built homes.
\end{abstract}

Keywords:

Passive house, environmental pollution, life-cycle assessment, certification systems for environmental quality, energy efficiency, ecological footprint and sustainability. 


\section{Resumen}

Ante la evidente crisis energética y la creciente contaminación ambiental, es imperante buscar alternativas para satisfacer las necesidades habitables y caloríficas del ser humano, enfocándonos principalmente en la energía natural más grande existente: el sol.

Las casas pasivas utilizan recursos de la naturaleza para obtener diferentes eficiencias e implementarlas de manera pasiva o transformándola por medio de tecnologías que funcionan en pro a las exigencias de los habitantes.

Las construcciones pasivas son diseñadas y construidas bajo parámetros establecidos por la

'Passivhaus' con la finalidad de ofrecer a los usuarios espacios habitables llevando de la mano considerables ahorros económicos en el importe energético, ofreciendo un ahorro anual significativo a comparación con viviendas construidas de manera convencional.

Palabras claves:

Casa pasiva, contaminación ambiental, ciclo de vida, huella ecológica y sustentabilidad.

\section{Introducción}

El término de viviendas sostenibles frecuentemente se relaciona con los aspectos proambientales, sin embargo, el término sostenible es mucho más extenso ya que contiene aspectos sociales, económicos, institucionales y gubernamentales. 1

Desde el punto de vista ambiental, uno de los principales propósitos de las edificaciones sostenibles es reducir sus impactos climáticos durante todo el su ciclo de vida, iniciando desde el diseño, el transcurso de construcción, hasta su mantenimiento y consumo día a día. 2

El objetivo dentro de la divulgación e aplicación de técnicas en el tema de viviendas pasivas es proporcionar a los usuarios un espacio confortable que brinde protección a los cambios de temperatura drásticos del exterior, donde se puedan desarrollar cada una de sus actividades otorgando satisfacción en sus necesidades de confort, reduciendo impactos negativos, además de que sea económicamente viable para los habitantes.

En la actualidad, se han promovido, a través de campañas de concientización ambiental constantemente, conceptos como eficiencia energética, sostenibilidad, impacto medioambiental, huella ecológica, entre otros, que a través de estos últimos años han ido cobrando relevancia.3

\footnotetext{
${ }^{1}$ La Vivienda Sustentable en México, (Metodología y Legislación). Grissel Hurtado López. UNAM, 2011

${ }^{2}$ Cambio climático: una visión desde México. México: Instituto Nacional de Ecología, Secretaría del Medio Ambiente y Recursos Naturales. Fernández A. Martínez J, 2004

${ }^{3}$ Huella ecológica y desarrollo sostenible, Juan Luis Doménech Quesada, AENOR, 2009.
} 
Arquitectura +

ISSN: 2518-2943

www.revistas.uni.edu.ni/index.php?jo

urnal=arquitectura

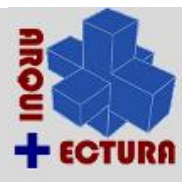

Vol. 3- Número 5 / Junio 2018

Viviendas pasivas a favor del medio ambiente

Ortiz Monroy Héctor / hector.ortiz@uaq.mx

* Quizamán Velasco Estefani Rosalía / equizaman@gmail.com

Por estas razones, el cambio climático siendo un factor transcendental a considerar durante el desarrollo y diseño de una casa pasiva -desde el punto de vista como constructores o diseñadores- se especulan los alcances que se pueden lograr con el objetivo de minimizar emisiones contaminantes, muchas veces desde la construcción y fabricación de materiales, como el dióxido de carbono, protegiendo con el tiempo la atmósfera y posteriormente hacer frente de manera principal el consumo energético; sin embargo, no solo la contaminación atmosférica es la que genera efectos sobre el medio ambiente, ahora bien, debemos considerar con la misma importancia la contaminación acústica, visual y olfativa que asimismo se presentan en los diferentes tipos de vivienda.

Para combatir estos factores se deben tomar como referencia diferentes estrategias con la finalidad de lograr una acción positiva al momento de diseñar una casa pasiva.

"Passivhaus es un estándar de calidad en torno a la eficiencia energética, que busca el mayor grado de confort térmico de los habitantes de un espacio; además, permite reducir significativamente el consumo de energía. Diversos estudios muestran que, a escala global, específicamente en Alemania, se reduce hasta 90 por ciento el uso de energía en comparación con una edificación convencional" (Egea 2015).

El concepto aplicado a las casas pasivas tiene la finalidad de reducir los consumos energéticos basados en:

- Aprovechamiento del calor solar.

- Aislamiento térmico en paredes, techos, pisos, puertas y ventanas.

- Hermeticidad en el aire.

- Sistema de ventilación muy eficaz, en el cual no se tengan pérdidas ganadas al interior de la vivienda.

- Aprovechamiento del calor procedente de los usuarios y electrodomésticos. 
Arquitectura +

ISSN: 2518-2943

www.revistas.uni.edu.ni/index.php?jo

urnal=arquitectura

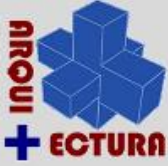

Vol. 3- Número 5 / Junio 2018

Viviendas pasivas a favor del medio ambiente

Ortiz Monroy Héctor / hector.ortiz@uaq.mx

* Quizamán Velasco Estefani Rosalía / equizaman@gmail.com

En la Figura 1 se muestran los puntos antes mencionados:

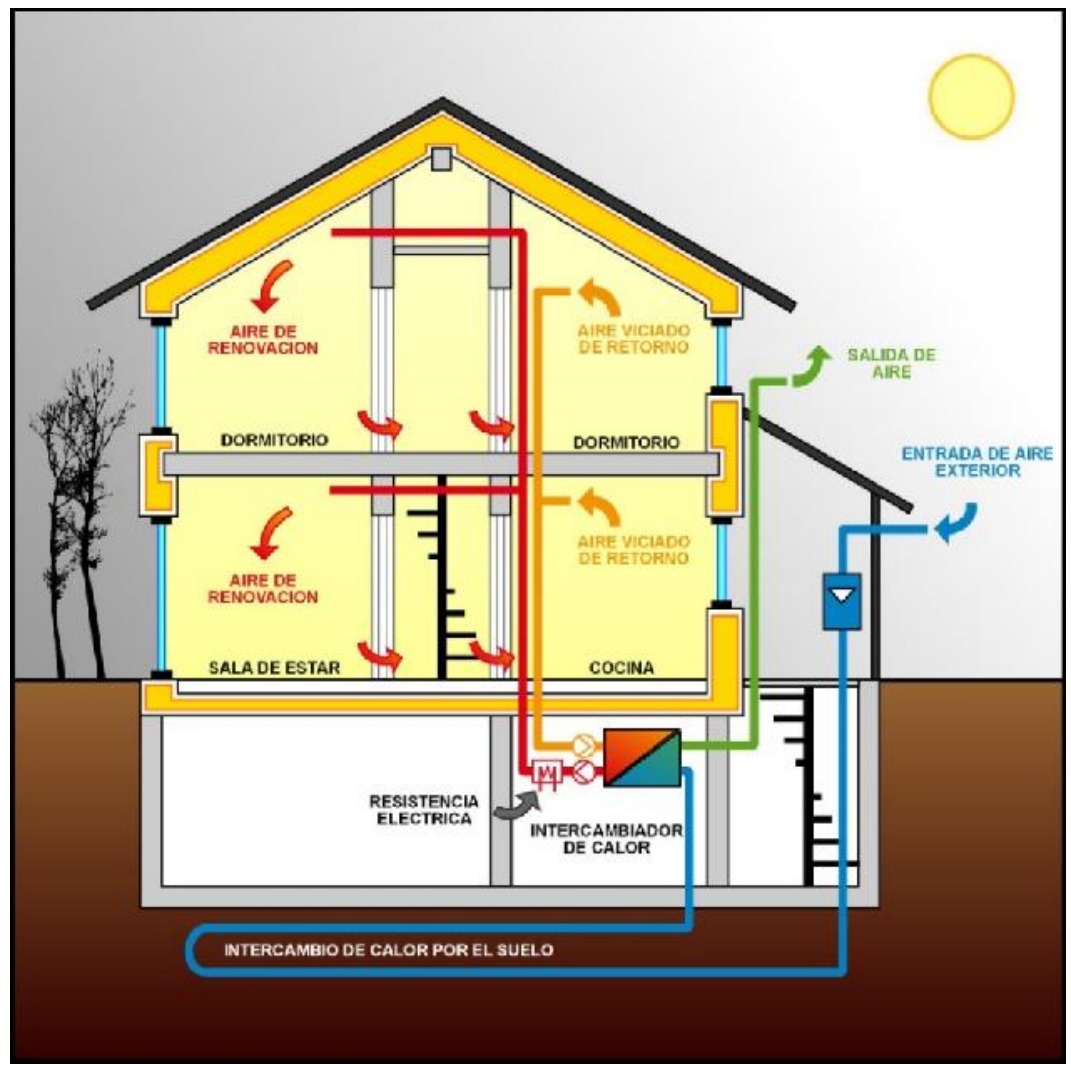

Fig. n 1. Funcionamiento de casas Passivhaus, sistema caracterizado por su eficiencia energética, confort y rentabilidad. ¿Cuál es la fuente de la imagen?

Con el conocimiento de los puntos antes mencionados, las casas pasivas se basan en un modelo de construcción, regidos por una serie de parámetros constructivos para el funcionamiento en conjunto, y cumplimiento de normas regulatorias que se puedan aplicar en cualquier proyecto a ejecutar.

La base de estas viviendas, además de generar menos insumos energéticos, tiene como regla primordial aprovechar al máximo las condiciones climáticas ofrecidas por la zona geográfica donde estén ubicadas, sirviendo esto como calefacción y refrigeración sin utilizar de manera excesiva recursos activos.

Así mismo, otro beneficio para las construcciones pasivas es que al momento de construir este tipo de las viviendas se pueden aprovechar los recursos naturales locales y aun así poder cumplir con las normas establecidas. 
Arquitectura +

ISSN: 2518-2943

www.revistas.uni.edu.ni/index.php?jo

urnal=arquitectura

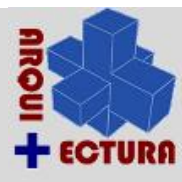

Vol. 3- Número 5 / Junio 2018

Viviendas pasivas a favor del medio ambiente

Ortiz Monroy Héctor / hector.ortiz@uaq.mx

* Quizamán Velasco Estefani Rosalía / equizaman@gmail.com

\section{Metodología}

El Instituto Passive House (PHI s.f.), surge en Alemania en el año 1988 como instituto independiente con la finalidad de desarrollar un estándar de construcción o readaptación para casas con consumos nulos de energía. (Gauna 2011).

Actualmente está comprobado que en Alemania las casas construidas bajo estos parámetros reducen un consumo de calefacción hasta un 85\% comparado con las construcciones tradicionales que no cuentan con los parámetros establecidos (aislantes, puentes térmicos ni ventanas con acristalamientos especiales) (IDAE 2016).

El objetivo principal de estas viviendas es que gracias al sol, al aislamiento, a los usuarios y a los electrodomésticos se puede mantener cierto grado de confort de manera pasiva (sin tener que recurrir a elementos activos -aparatos eléctricos-).

La Plataforma de Edificación Passivhaus (PEP s.f.), establecida en España, se encarga entre otras cosas- de la difusión sobre las múltiples ventajas de las casas construidas o adaptadas bajo los parámetros para la creación de una vivienda con sistemas pasivos de climatización, el cual estima es posible disminuir hasta un 70\% de energía anualmente (Gauna 2011).

Las casas pasivas se rigen bajo el siguiente método, atacando de manera puntual los siguientes principios básicos, tal como se muestra en las siguientes figuras.

1. Diseño.

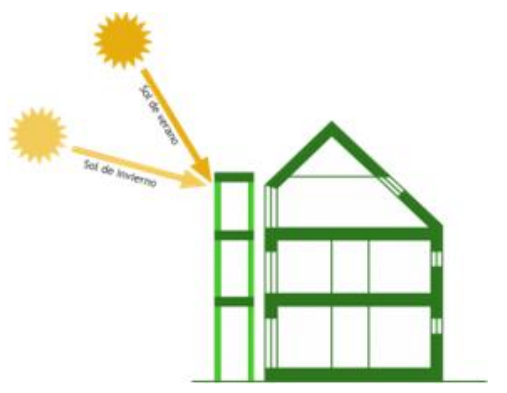

Es importante considerar todos los recursos naturales -orientación y vientos dominantes- geo localizados donde se vaya a ubicar la vivienda, ya que estos funcionan con una perfecta combinación entre los elementos térmicos de los envolventes, orientación, captación y protección solar.

Figura 2. Criterios de diseño de una Passivhaus. Fuente: Energiehaus Edificios Pasivos

2. Aislamiento térmico en muros y cubiertas. 
Arquitectura +

ISSN: 2518-2943

www.revistas.uni.edu.ni/index.php?jo

urnal=arquitectura

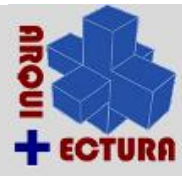

Vol. 3- Número 5 / Junio 2018

Viviendas pasivas a favor del medio ambiente

Ortiz Monroy Héctor / hector.ortiz@uaq.mx

* Quizamán Velasco Estefani Rosalía / equizaman@gmail.com

Mientras tanto es fundamental cumplir con un buen aislante térmico para logar un óptimo

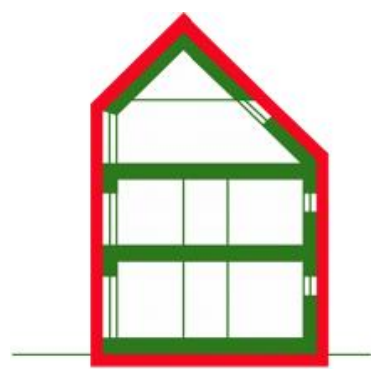
funcionamiento sobre el cúmulo de requerimientos de una casa

pasiva, ya que el aislamiento es crucial a la hora de consumir energía, es por eso que la selección de materiales nos ayudará a obtener un resultado favorable, donde el intercambio de calor entre el interior y el exterior sea solamente por inercia.

Figura 3. Aislamiento térmico continúo. Fuente: Energiehaus Edificios Pasivos

favorable para el aprovechamiento de radiación solar ventilación.

3. Orientación

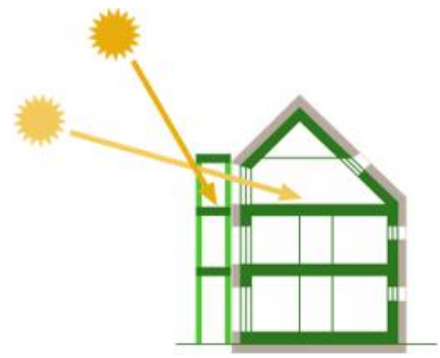

Es importante que optimicemos las orientaciones en beneficio de la vivienda ya que se puede aprovechar mejor la luz natural durante el día y lograr mayor captación solar para que cuando baje la temperatura, el calor ganado durante el día haga su trabajo en el interior de la vivienda.

Figura 4. Utilización y aprovechamiento de orientaciones para captar radiación solar. Fuente: Energiehaus Edificios Pasivos.

4. Eliminación de puentes térmicos

Aquellas zonas donde la vivienda tiene debilidades por distintos factores -cambios de

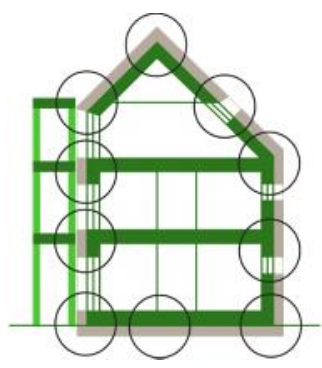
materiales, uniones de muros, ventanas-son sitios que fácilmente trasladan frio y humedad del exterior al interior. En este caso, como su nombre lo dice, es elemental no omitir este tipo de detalles ya que se corre el riesgo de perder la energía ganada con las estrategias previas.

5. Estanqueidad del aire y ventilación con recuperación de calor.

Figura 5. Puentes térmicos. Fuente: Energiehaus Edificios Pasivos. 
Arquitectura +

ISSN: 2518-2943

www.revistas.uni.edu.ni/index.php?jo

urnal=arquitectura

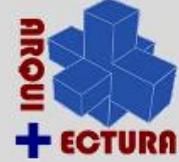

Vol. 3- Número 5 / Junio 2018

Viviendas pasivas a favor del medio ambiente

Ortiz Monroy Héctor / hector.ortiz@uaq.mx

* Quizamán Velasco Estefani Rosalía / equizaman@gmail.com

La hermeticidad del aire nos ayuda a mantener confort térmico en el interior de la vivienda, ya que el cruce de aire crea cambios bruscos de temperatura provocando temperaturas no

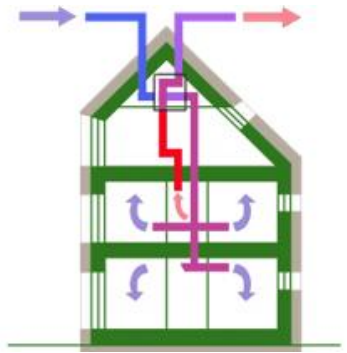
deseadas, por este motivo la ventilación mecánica controlada se encargará de regular este movimiento e intercambio de aire con el exterior, en el cual el aire frío saldrá por medio de extractores y el aire nuevo pasará por un filtro que además de eliminar partículas nocivas de contaminación entrará con temperatura regulada.

Figura 6. Funcionamiento ventilación mecánica. Fuente: Energiehaus Edificios Pasivos.

6. Puertas y ventanas.

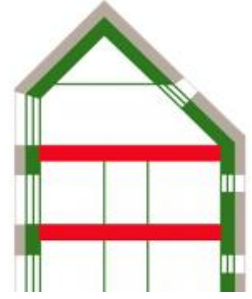

Estos elementos son los más débiles de la envolvente de una vivienda, es por eso que además de tener una doble función es necesario contar con un flujo térmico que no permita perdidas, debe permitir tener ganancias solares, sobre todo en épocas de invierno.

_Figura 7. Aislantes térmicos. Fuente: Energiehaus Edificios Pasivos.

En relación con los parámetros antes descritos, se puede intervenir de una manera sustentable en el diseño y aprovechamiento de recursos que no afecten severamente el medio ambiente.

Ahora bien, por lo que corresponde a la generación, construcción y funcionamiento de viviendas se contribuye significativamente en el cambio climático creando excesivo $\mathrm{CO} 2$, ya que en México el $16.2 \%$ del consumo energético es ocasionado por las viviendas, generando alrededor de $4.9 \%$ de emisiones contaminantes.4

Con respecto a la generación de CO2, en el año 2011 nuestro país contribuyó con $1.4 \%$ de las emisiones globales, ubicándose como el décimo segundo país con mayores emisiones del mundo (DOF 2013).

Sin embargo, el incremento de la demanda energética, que consecuentemente irá generando más emisiones, ampliando la vulnerabilidad al cambio climático, dadas las condiciones expuestas anteriormente.

\footnotetext{
${ }^{4}$ Boletín 71. CONAVI. México reducirá el 100\% de emisiones de CO2 con vivienda NAMA. https://www.gob.mx/conavi/prensa/boletin-71-mexico-reducira-el-100-de-emisiones-de-co2-con-viviendanama?idiom $=\mathrm{es}$
} 
Arquitectura +

ISSN: 2518-2943

www.revistas.uni.edu.ni/index.php?jo

urnal=arquitectura

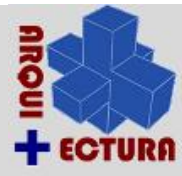

Vol. 3- Número 5 / Junio 2018

Viviendas pasivas a favor del medio ambiente

Ortiz Monroy Héctor / hector.ortiz@uaq.mx

* Quizamán Velasco Estefani Rosalía / equizaman@gmail.com

Por otro lado, el estudio en distintas perspectivas para combatir este fenómeno influye de manera territorial, llevando de la mano juicios económicos. Pese a que, la demanda energética se adopta de diversas modalidades y depende directamente de la demanda de cada usuario, vale destacar que la emisión de gases está proporcionalmente vinculado con la condición social de los habitantes mediante el gusto, usos y preferencias que se desarrollan en su residencia.

En relación al costo-beneficio de pretender conservar y reducir la generación de CO2, los principios básicos mencionados anteriormente, aplicados en el diseño y construcción de una vivienda trabajando en conjunto crean una casa pasiva.

Considerando el enfoque planeado por el Passive House Institute (PH por sus siglas en inglés), con el sistema constructivo planteado en las casas pasivas, éstas ahorran gran cantidad de energía por todas las ganancias solares que se logran día a día, en el que el egreso monetario de energía para la aplicación de calefacción y refrigeración es aún menor y se ve reflejada en la creación de los CO2.

Como se menciona anteriormente, este ahorro de energía disminuye directamente el impacto de las viviendas en la atmósfera a través de emisiones contaminantes para el medio ambiente.

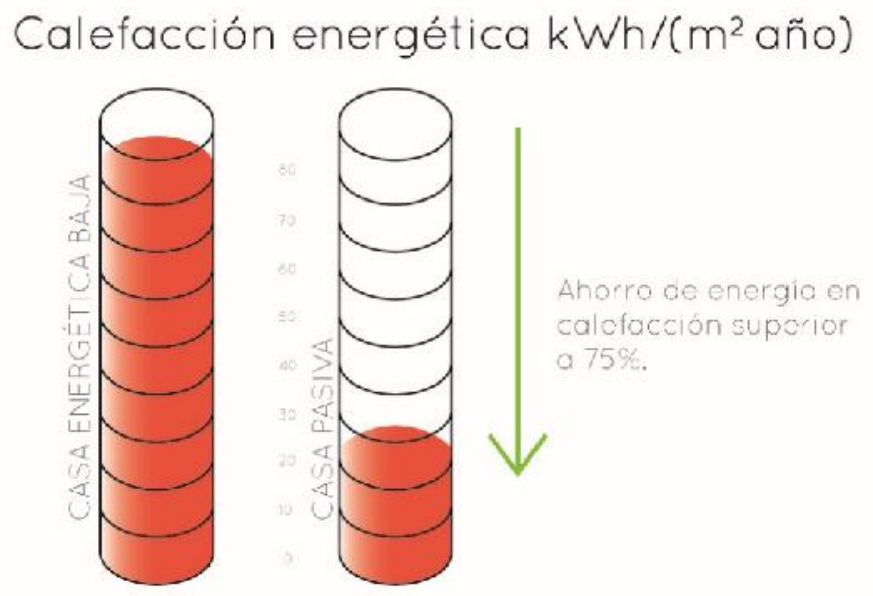

Ilustración 8 Ahorro de energía de calefacción entre un edificio de casa pasiva en comparación con una casa de baja energía. Fuente: Passive House Institute. 
Arquitectura +

ISSN: 2518-2943

www.revistas.uni.edu.ni/index.php?jo

urnal=arquitectura

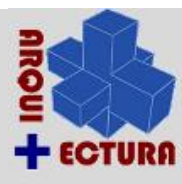

Vol. 3- Número 5 / Junio 2018

Viviendas pasivas a favor del medio ambiente

Ortiz Monroy Héctor /

hector.ortiz@uaq.mx

* Quizamán Velasco Estefani Rosalía / equizaman@gmail.com

En España, los edificios construidos bajo los parámetros Passivhaus, tienen un sobre costo por encima de las convencionales entre un $4 \%$ y $15 \%$, sin embargo cada vivienda tiene variables diferentes los cuales determinarán un valor exacto al costo final de la obra. ${ }^{5}$

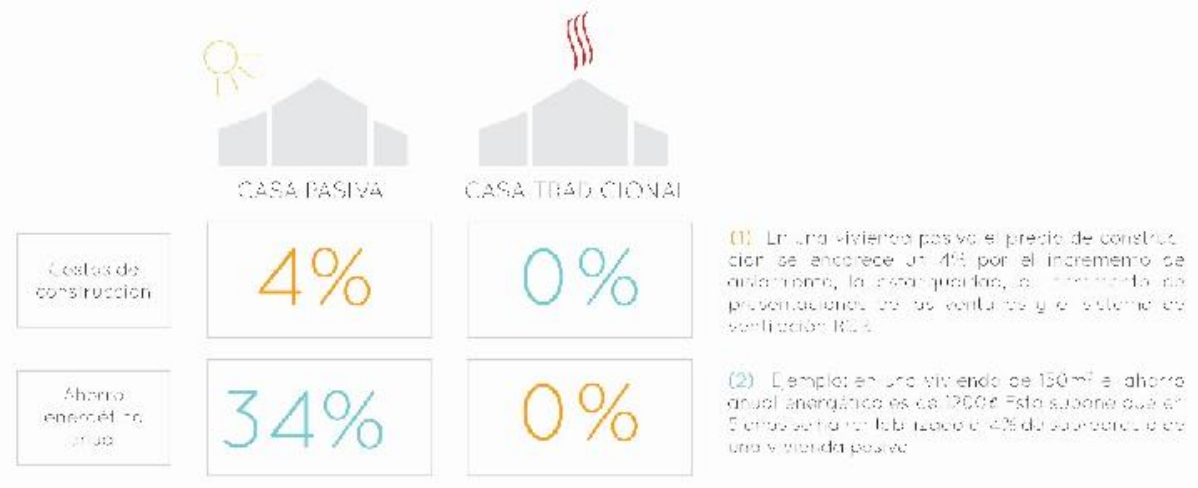

Figura 9. Comparación sobre el diseño de una vivienda pasiva contra una vivienda convencional. Fuente:

Somos Passivhaus. https://somospassivhaus.es/casas-pasiva.

\section{Conclusiones}

A partir de la revisión y valoración de las variables socioeconómicas dentro del consumo energético, es posible entender que al plantear y desarrollar proyectos ambientales eficientes, encontramos múltiples opciones para poder lograrlo.

Al considerar aportar y reducir emisiones en el país, es importante tomar en cuenta desde un inicio a contribuir de manera más puntual sobre el problema de contaminación ambiental, es por eso que desde el proceso de diseño arquitectónico, se requiere implementar medidas que contribuyan a una reducción de emisiones considerando los puntos o elementos a utilizar desde la construcción de cualquier edificación.

La arquitectura debe ser planteada y pensada de una manera para que desde su construcción pueda ser sostenible y como ejecutantes aplicar e influir en los usuarios, de tal modo que al habitarla puedan albergar y ofrecer el máximo confort, sin perjudicar al medio ambiente, resultando en una generación moderada de CO2.

Los objetivos de ataque como usuarios y ejecutantes pueden parecer abrumadores, y este artículo toca al menos un punto de partida de temas involucrados en este reto de participación a nivel vivienda a favor del uso y eliminación de elementos no sustentable.

\footnotetext{
${ }^{5}$ ¿Qué sobrecoste tiene una Passivhaus? Fuente: Energiehaus Edificios Pasivos. http://www.energiehaus.es/passivhaus/preguntas-mas-frecuentes/\#que-beneficios-tiene-vivir-en-una-passivhaus
} 
No obstante reside en cada individuo aplicar sus habilidades y conocimientos, contribuyendo con su experiencia, a obtener un efecto positivo sobre el desarrollo de viviendas sustentables, que además del cumplimiento con obligaciones federales por medio de normativas, también tenemos obligaciones sociales y morales, que como seres humanos podemos contribuir y aportar a este tipo de cuestiones.

Las edificaciones al crear ciudades han contribuido de manera considerable con los problemas ambientales, sin embargo, ahora pueden ser parte de la solución mediante el uso de las regulaciones basadas en los lineamientos de las casas pasivas perfilados a consumos casi nulos de energía generando muy bajas emisiones de $\mathrm{CO} 2$, conlleva a la conservación del planeta, optimizando la calidad de vida de los usuarios y otorgando confort interior.

\section{Referencias}

1. Brown, Dr Sarah Price and Helen. "Whole-life costs of a Passivhaus." Encraft, 2014: 9.

2. DOF, Diario Oficial de la Federación. "Acuerdo por el que se expide la Estrategia Nacional de Cambio Climático." M, México, 06 03, 2013.

3. DW-WORLD. "www.dw.com." 09 21, 2007. http://www.dw.com/es/casas-que-protegenel-medio-ambiente/a-2791696.

4. Egea, Marisa. La arquitectura pasiva reclama su lugar en México. INHAB, 2015.

5. Gauna, Javier Crespo Ruiz de. Guía del estándar Passivhaus. Edificios de consumo energético casi nulo. Madrid: Zehnder, 2011.

6. IDAE. III Congreso Edificios Energía Casi Nula. Madrid: Grupo Tecma Red S.L, 2016.

7. Lezama, José Luis. La construcción social y política del medio ambiente, México. El Colegio de México, 2004.

8. PEP. "Plataforma de Edificación Passivhaus." n.d.

9. PHI. "Institute Passive House." n.d.

10. Quesada, Juan Luis Doménech. Huella ecológica y desarrollo sostenible. AENORediciones, 2009.

11. Rosas, María Cristina. "Muy Interesante." http://www.muyinteresante.com.mx. Diciembre 11 2017. http://www.muyinteresante.com.mx/tecnologia/14/12/11/generacion-energiasrenovables-mexico/.

12. Sánchez, Landy. "El consumo energético de los hogares en México." 2016. http://www.somede.org/coyuntura-demografica/articulos/sanchez-20120716.pdf.

13. Spirtz, Stephan. Casas que protegen el medio ambiente. DW, 2007.

14. Wassouf, Micheel. De la casa pasiva al estándar Passivhaus. Barcelona: Gustavo Gili, 2014. 